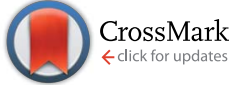

Cite this: RSC Adv., 2014, 4, 53265
Received 3rd July 2014

Accepted 7th October 2014

DOI: $10.1039 / c 4 r a 06620 k$

www.rsc.org/advances

\section{Insight into magnetite nanoparticle phase evolution in solvothermal synthesis through a simple method based on iron chloride and metallic iron}

\begin{abstract}
F. B. F. Silva, ${ }^{a}$ E. C. Paris, ${ }^{b}$ G. M. da Costa $^{c}$ and C. Ribeiro ${ }^{\star b}$
This paper presents the development and optimization of the synthesis of magnetic nanoparticles composed of iron oxides by a solvothermal method with benzyl alcohol using iron(III) chloride hexahydrate as a metallic precursor in the substitution of iron(III) acetylacetonate. The synthesis parameters were studied and varied to obtain a high yield of magnetite. Metallic iron was evaluated as a reducing agent and iron source. Furthermore, the use of urea as a precipitation agent was crucial to increasing the yield of magnetite, at once reducing the acidity of the medium and inducing the precipitation of $\mathrm{Fe}^{2+}$ ions. Longer treatment times and no stirring led to an increase in the yield of magnetite, whose formation process followed the Ostwald step rule. The particles were characterized by X-ray diffraction, Mössbauer spectroscopy, FTIR, TEM, SEM-FEG and VSM (Vibrating Sample Magnetometry). The phases were quantified by the Rietveld method and Mössbauer spectroscopy, and a $97 \%$ maximum yield of magnetite was achieved. The polymerization of benzyl alcohol during the solvothermal treatment was shown to play an important role in the nanoparticle capping. The polymerized resin was characterized by FTIR and NMR- ${ }^{1} \mathrm{H}$, and a mechanism for the polymerization reaction was proposed to elucidate the synthetic route and its relationship to particle formation and stability.
\end{abstract}

\section{Introduction}

Recent advances in colloidal synthesis have allowed for the exploration and manipulation of the magnetic properties of solid materials at the nanometer scale and enabled a range of novel applications of great interest to researchers from several disciplines, including magnetic fluids, catalysis, biotechnology/ biomedicine, magnetic resonance imaging, data storage and environmental remediation. ${ }^{\mathbf{1 - 3}}$ Magnetic nanoparticles, when functionalized, can be very promising for applications in which, being so small and magnetically separable, the particles may act as quasi-homogeneous systems, combining high reactivity, high dispersion and easy separation. These features are especially interesting in catalysis, and many publications have described the application of such magnetic nanoparticles in facilitating the effective separation and recycling of expensive catalysts. ${ }^{\mathbf{4}}$ Certain technological enzymatic processes can also benefit by the functionalization of magnetic nanoparticles with

${ }^{a}$ Departamento de Quimica - UFSCar, Rod. Washington Luiz, km235, CEP 13565-905 São Carlos, SP, Brazil

${ }^{b}$ Embrapa Instrumentação, Rua XV de Novembro, 1452, CEP 13560-970, São Carlos, SP, Brazil. E-mail: caue.ribeiro@embrapa.br

${ }^{c}$ Departamento de Química - Universidade Federal de Ouro Preto, CEP 35400-000, Ouro Preto, $M G$, Brazil enzymes, at once enabling the recovery and the reuse of soluble enzymes. $^{5-7}$

Iron oxide nanoparticles are most likely the best-studied magnetic nanomaterial due to their nontoxicity and biocompatibility. Nevertheless, the formation process of these magnetic nanoparticles is still not clear for many reaction systems. ${ }^{\mathbf{8}-10}$ New strategies for synthesizing these particles by controlling their shape and size (and surface) and for protecting them against oxidation are fundamental to the use of these materials in a wide range of specific applications, especially on a large scale. These limitations can be overcome by nonaqueous approaches, which provide a more effective control of size distribution due mainly to the moderate reactivity of the oxygen-carbon bond, leading to slower reaction rates, and to the surfactant role play by certain organic solvents. ${ }^{\mathbf{1 0 - 1 2}}$ For example, the thermal decomposition of organometallic or metal organic iron precursors such as iron(III) cupferronate $\left(\mathrm{FeCup}_{3}\right)$, iron(III) acetylacetonate $\left(\mathrm{Fe}(\mathrm{acac})_{3}\right)$ and pentacarbonyl iron $\left(\mathrm{Fe}(\mathrm{CO})_{5}\right)$ in high-boiling organic solvents containing stabilizing surfactants leads to a very narrow size distribution of small magnetite or maghemite nanoparticles. ${ }^{13-19}$ Pinna et al. (2005) described a surfactant-free route to synthesizing magnetite nanoparticles by using benzyl alcohol as a solvent and a ligand at the same time to overcome the drawbacks of surfactant- 
controlled approaches. The direct reaction between benzyl alcohol and iron(III) acetylacetonate under solvothermal conditions has been observed to produce high-purity magnetite nanocrystals with sizes ranging from 12 to $25 \mathrm{~nm} .{ }^{20}$ The "benzyl alcohol route" has proven to be a suitable strategy for synthesizing several binary metal oxide nanoparticles, perovskites and hybrid materials. ${ }^{21-23}$ This simple and robust method leads to low amounts of organic impurities in the products, therefore making it suitable for studying nanoparticle formation. ${ }^{\mathbf{1 1}}$

The "benzyl alcohol route" has been applied to synthesize iron oxide nanoparticles using mainly iron(III) acetylacetonate as a precursor; however, common precursors employed in solgel synthesis, such as $\mathrm{Fe}(\mathrm{III})$ and $\mathrm{Fe}(\mathrm{II})$ chlorides, nitrates, sulfates or even metallic iron, have not been reported in the literature as possible precursors for this method. ${ }^{8}$ This fact suggests that the phase evolution in the aforementioned system is still poorly understood, despite several other oxides having been synthesized using metal halides..$^{24,25}$

In this work, we report the synthesis of magnetite nanoparticles following the benzyl alcohol route by using iron(III) chloride hexahydrate and metallic iron as iron precursors to investigate the mechanisms involved in $\mathrm{Fe}_{3} \mathrm{O}_{4}$ phase evolution under solvothermal conditions. Other processes involved in the synthesis, such as the polymerization of benzyl alcohol, were also investigated to understand their role in the phase formation and stability of the products.

\section{Experimental}

All materials were prepared under solvothermal conditions with different proportions of iron chloride hexahydrate $\left(\mathrm{FeCl}_{3} \cdot 6 \mathrm{H}_{2} \mathrm{O}\right)$ and metallic iron $\left(\mathrm{Fe}^{0}\right)$ while varying the synthetic parameters (temperature, time, stirring and addition of urea). In a typical synthesis procedure, carried out in a glovebox $\left(\mathrm{H}_{2} \mathrm{O}<0.1 \mathrm{ppm}\right)$, the iron precursors (totaling $4.5 \mathrm{mmol}$ of iron) were added to 30 $\mathrm{mL}$ of benzyl alcohol, containing $1.0 \mathrm{~g}$ of powdered urea (16.6 $\mathrm{mmol}$ ). A similar experiment was performed without the addition of urea. The mixtures were homogenized in a borosilicate glass cup with an inner volume of $60 \mathrm{~mL}$, under magnetic stirring, and then transferred to a stainless steel solvothermal reactor that was carefully sealed. The apparatus was removed from the glovebox and heated to $250{ }^{\circ} \mathrm{C}$ for 24,48 and 96 hours. The materials obtained after the solvothermal treatment were cooled to room temperature, centrifuged and thoroughly washed with acetone after sonication in an ultrasonic bath for 3 minutes. This procedure was performed to remove the organic residues on the particles' surface. A second washing step with ethanol was necessary when urea was added to the reaction mixture to remove the ammonium chloride byproduct. After the washing steps, the precipitates were dried in air at $70{ }^{\circ} \mathrm{C}$.

The particles were structurally characterized in an X-ray diffractometer (Shimadzu XRD 6100) with CuK $\alpha$ radiation, using a $\theta-2 \theta$ configuration, at $30 \mathrm{kV}$ and $30 \mathrm{~mA}$. The $2 \theta$ range used in the measurement was $10-110^{\circ}$, with a step size of $0.02^{\circ}$ and a step time of $4.0 \mathrm{~s}$. Quantitative analysis by Rietveld refinement was performed with GSAS software. ${ }^{26}$ Mössbauer spectra (MS) were obtained at room temperature with a spectrometer using a constant-acceleration drive with a triangular reference signal and 1024 channels and in the velocity range of -11 to $11 \mathrm{~mm} \mathrm{~s}^{-1}$ (increment of $\sim 0.046 \mathrm{~mm} \mathrm{~s}^{-1}$ ). The velocity was calibrated from the MS of a standard alpha-Fe foil at room temperature. The spectra were computer-fitted either with discrete Lorentzian sextets or doublets.

The sample size and morphologies were examined by field emission gun-scanning electron microscopy (FEG-SEM) in a JEOL JSM6701F microscope operating at $5.0 \mathrm{kV}$ and by transmission electron microscopy (TEM) in a Philips CM-120 microscope (operating at $120 \mathrm{kV}$ ) through bright field (BF), dark field (DF) images and electron diffraction (ED) of selected particles. The TEM samples were dispersed in ethanol, and a few drops of the samples were deposited on a cooper-carbon grid. Hysteresis loops of the samples were obtained by vibrating sample magnetometry, performed at room temperature with a vibrating sample measurement (EG\&G Princeton Applied Research equipment) in a home-made setup.

Fourier-transform infrared spectroscopy (FTIR) was performed in a Bruker/Equinox 55 instrument, from 4000 to 400 $\mathrm{cm}^{-1}$, by conducting an average of 100 scans and using transmission and diffuse-reflectance modes. To analyze the eventual polymerization of the solvent (benzyl alcohol), high-resolution ${ }^{1} \mathrm{H}$ nuclear magnetic resonance spectroscopy (Bruker $600 \mathrm{MHz}$ equipment, operating under $14.1 \mathrm{~T}$ ) was performed. The chemical shift values were calibrated using tetramethylsilane as a reference. To identify the shifts in a proposed molecular structure, a simulation was performed using the NMR-Predictor program (available at http://www.nmrdb.org/).

\section{Results and discussion}

Because in magnetite $\mathrm{Fe}^{\mathrm{III}}$ and $\mathrm{Fe}^{\mathrm{II}}$ species are present, the syntheses were initially carried out using different proportions of $\mathrm{FeCl}_{2}$ and $\mathrm{FeCl}_{3}$, under similar solvothermal conditions. However, in all cases, the formation of hematite as a major phase was observed, and only small amounts of magnetite were obtained. These results indicated that a redox pair would be necessary to achieve the proper phase formation. Thus, we opted to introduce metallic iron $\left(\mathrm{Fe}^{0}\right)$ as a reductant, aiming to



Fig. 1 XRD patterns of the particles synthesized with different amounts of metallic iron. 
reduce $\mathrm{Fe}^{3+}$ ions during the solvothermal process because $\mathrm{Fe}^{0}$ can act as an iron precursor and reducing agent. ${ }^{27}$ Fig. 1 shows the XRD patterns obtained for different $\mathrm{Fe}^{0} / \mathrm{Fe}^{3+}$ molar ratios, at $250{ }^{\circ} \mathrm{C} / 24 \mathrm{~h}$ under stirring. All diffractograms show a mixture of hematite and magnetite, except the one correspondent to $100 \%$ metallic iron, in which the only observed phase is the metallic iron. These results indicate that the solvent by itself, even at high temperature and pressure, does not play an oxidative role during the synthesis procedures. It also shows that $\mathrm{Fe}^{0}$ does in fact interact with $\mathrm{Fe}^{3+}$ during the redox process. It was possible to observe the dependence of the magnetite yield on the amount of metallic iron. This relationship could be easily observed by comparing the relative intensity between the peaks at $33.2^{\circ}$, the most intense peak of hematite, and $35.6^{\circ}$, the most intense peak of magnetite. The best result was reached with $25 \%$ of metallic iron, indicating an optimum range for phase formation. In an attempt to improve the magnetite yield, urea $\left(\mathrm{CO}\left(\mathrm{NH}_{2}\right)_{2}\right)$ was added to provide a basic medium, adequate for the formation of magnetite phase because $\mathrm{Fe}(\mathrm{OH})_{2}$, the most probable precursor of $\mathrm{Fe}^{\mathrm{II}}$, is less soluble under this condition. $\mathrm{FeCl}_{3} \cdot 6 \mathrm{H}_{2} \mathrm{O}$ is a fairly strong Lewis acid, and the stability of $\mathrm{Fe}(\mathrm{OH})_{2}$ depends on a source of $\mathrm{OH}^{-}$, which can be obtained by the decomposition of urea. ${ }^{27-29}$

As expected, magnetite production was significantly improved (Fig. 2a). Furthermore, Tamaura suggested that $\mathrm{Cl}^{-}$ retards magnetite formation by hindering the condensation of neighboring $\mathrm{OH}$ groups and consequent formation of $\mathrm{Fe}-\mathrm{O}-\mathrm{Fe}$ linkages. Thus, this contaminant can be easily separated because $\mathrm{NH}_{4}{ }^{+}$ions formed during the decomposition of urea are able to capture $\mathrm{Cl}^{-}$ions by forming $\mathrm{NH}_{4} \mathrm{Cl}$, which is insoluble in the reaction medium. ${ }^{30}$

Having established the optimum metallic iron fraction to be $25 \%$ and adding an excess of urea, the reaction time was extended from 24 (Fig. 2a) to 48 (Fig. 2b) to $96 \mathrm{~h} \mathrm{(Fig.} \mathrm{2c).} \mathrm{These}$ figures clearly show the increase in the yield of magnetite, indicating the beneficial effect of longer reaction times. It can also be observed that the calculated XRD lineshapes resulting from the Rietveld refinement closely match the experimental data. Considering the absence of amorphous phases and the existence of only hematite and magnetite, a quantitative analyses was performed, the results of which are presented in Table 1. It is noteworthy that for longer periods, the magnetite yield was higher than $80 \%$, showing that the formation of hematite most likely preceded that of magnetite. Additionally, the role of $\mathrm{Fe}^{0}$ as a reductant species was also confirmed. However,

Table 1 Phase quantification (weight\%) obtained by Rietveld refinements and Mössbauer spectroscopy

\begin{tabular}{|c|c|c|c|c|c|}
\hline & \multicolumn{2}{|c|}{ Rietveld } & \multicolumn{3}{|c|}{ Mössbauer } \\
\hline & $\mathrm{Fe}_{3} \mathrm{O}_{4}$ & $\mathrm{Fe}_{2} \mathrm{O}_{3}$ & $\mathrm{Fe}_{3} \mathrm{O}_{4}$ & $\mathrm{Fe}_{2} \mathrm{O}_{3}$ & $\mathrm{FeO}(\mathrm{OH})$ \\
\hline u25\%_24 h_s & 61.9 & 38.1 & 60.9 & 35.4 & 3.6 \\
\hline u25\%_48 h_s & 76.0 & 24.0 & 76.5 & 19.5 & 4.0 \\
\hline u25\%_96 h_s & 83.5 & 16.5 & - & - & - \\
\hline u25\%_96 h_ns & 95.8 & 4.2 & 90.0 & 5.0 & 5.0 \\
\hline u12\%_96 h_ns & 97.4 & 2.6 & 96.0 & 1.0 & 3.0 \\
\hline
\end{tabular}
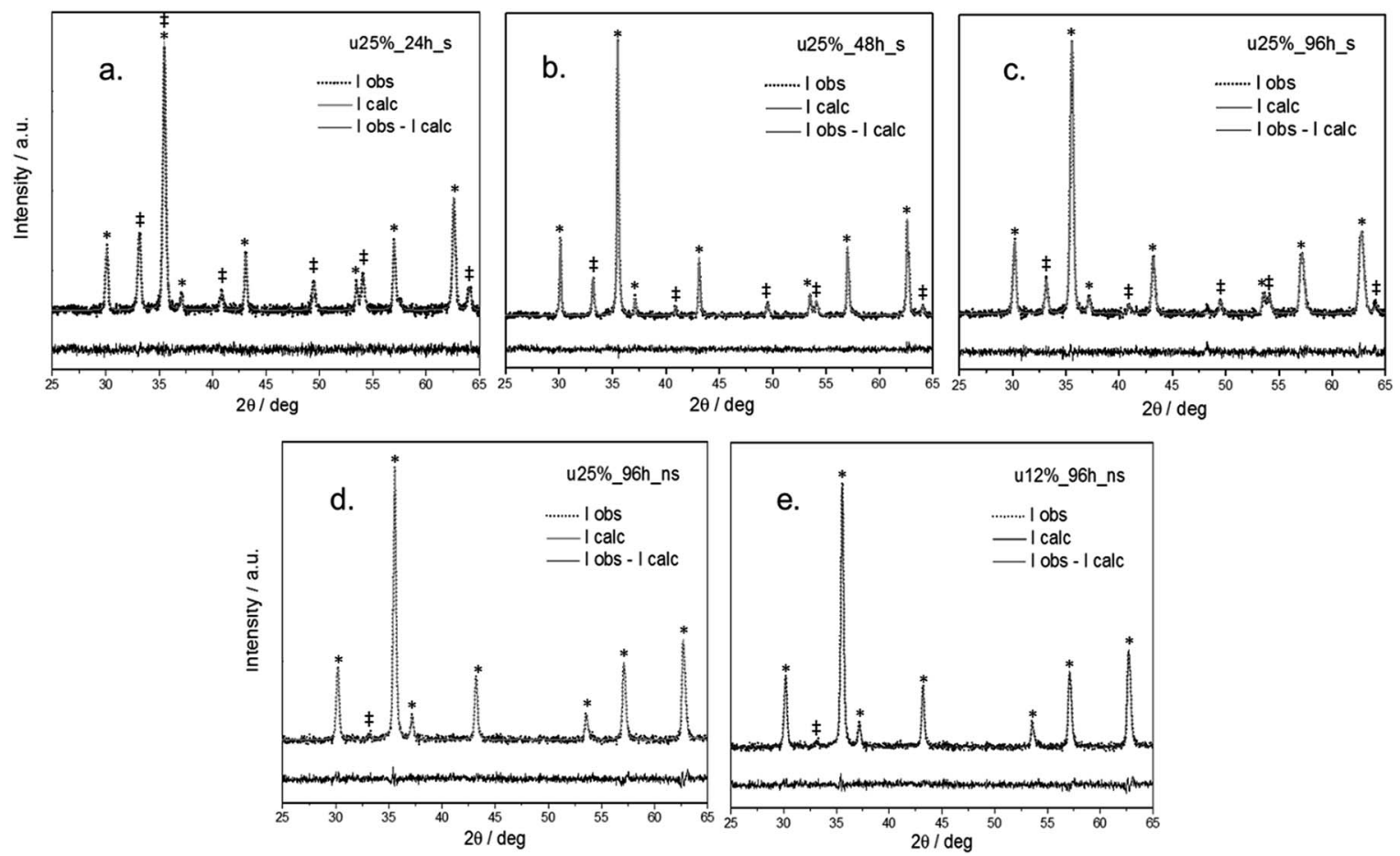

Fig. 2 Rietveld refinement plots of the samples synthesized with urea under various conditions: (a) $25 \% \mathrm{Fe}^{0}$ for 24 hours; (b) $25 \% \mathrm{Fe} \mathrm{for}^{0} 88$ hours; (c) $25 \% \mathrm{Fe}^{0}$ for 96 hours; (d) $25 \% \mathrm{Fe}^{0}$ for 96 hours and no stirring; (e) $12 \% \mathrm{Fe}^{0}$ for 96 hours and no stirring. Diffraction peaks assigned to hematite phase are marked with double dagger symbols $(\$)$, and magnetite phase are marked with an asterisk (*). 
because stirring may interfere with the phase formation, this parameter was also evaluated. The magnetite yield for the sample prepared with $25 \% \mathrm{Fe}^{0}$ for 96 hours and no stirring (Fig. 2d) was improved to 96\%, as shown in Table 1.

This improvement can be explained by considering that stirring can induce the agglomeration of as-formed by magnetic interactions, reducing the total effective area for reaction and leading to possible segregation. Additionally, the residual hematite content may be related to an excess of $\mathrm{Fe}^{0}$ in the reaction. In fact, when performing the same experiment but using $12 \% \mathrm{Fe}^{0}$ instead of $25 \% \mathrm{Fe}^{0}$, (Fig. 2e) we observed a slight increase in the magnetite yield to $97.4 \%$. Thus, the optimum $\mathrm{Fe}^{0}$ content appears to be between $12 \%$ and $25 \%$.
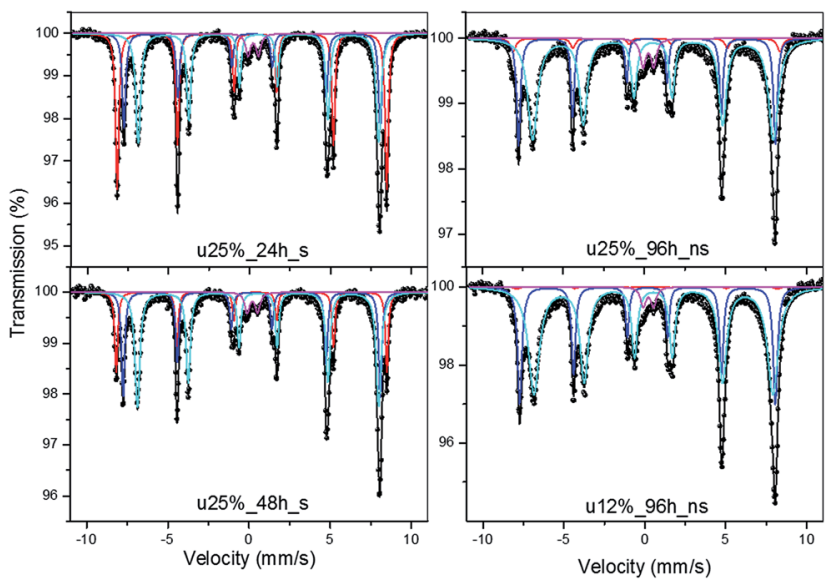

Fig. 3 Mössbauer spectra and their curve-fitting results for the samples u25\%_24 h_s, u25\%_48 h_s, u25\%_96 h_ns and u12\%_96 h_ns.
Magnetite and hematite can be satisfactorily distinguished by their XRD patterns. On the other hand, magnetite and maghemite have very similar patterns and cannot be distinguished by this technique. Therefore, Mössbauer spectroscopy performed at room temperature was used to elucidate the true composition of these magnetic particles. The fitted spectra are shown in Fig. 3, whereas the calculated amounts of magnetite and hematite are presented in Table 1 . The spectrum of all samples showed magnetite contents ranging from 60.9 to $96.0 \%$, values that are in a good agreement with the results of the Rietveld method (see Table 1). However, the relative area ratios of the two magnetite sextets deviate substantially from the ideal value of 1.88 for pure magnetite. This finding can be attributed either to the presence of non-stoichiometric magnetite or to the presence of some maghemite. The presence of a low-intensity doublet may be correlated to a small amount of goethite.

Fig. 4a to c show BF-TEM images of selected samples, which indicate particle sizes ranging from 50 to $100 \mathrm{~nm}$. Also, from a representative zoom out FEG-SEM image (Fig. 4d) it was possible to analyze the particle size by direct image analysis of more than 200 particles. The results are shown in a histogram (Fig. 4e), confirming the average diameter of $92.8 \mathrm{~nm}$. At these sizes, it is expected that the magnetite phase would be more stable, in accord with the results of other characterizations. The sample synthesized over a period of 96 hours showed higher agglomeration and size dispersion, which is expected since longer treatment times would lead to grain growth. Furthermore, under this condition, higher particle sizes may alter the magnetic behavior from superparamagnetism to ferrimagnetism, leading to permanent magnetization.

Fig. 5 shows a DF-TEM image and ED for the sample synthesized in 96 hours, where the diffraction pattern is

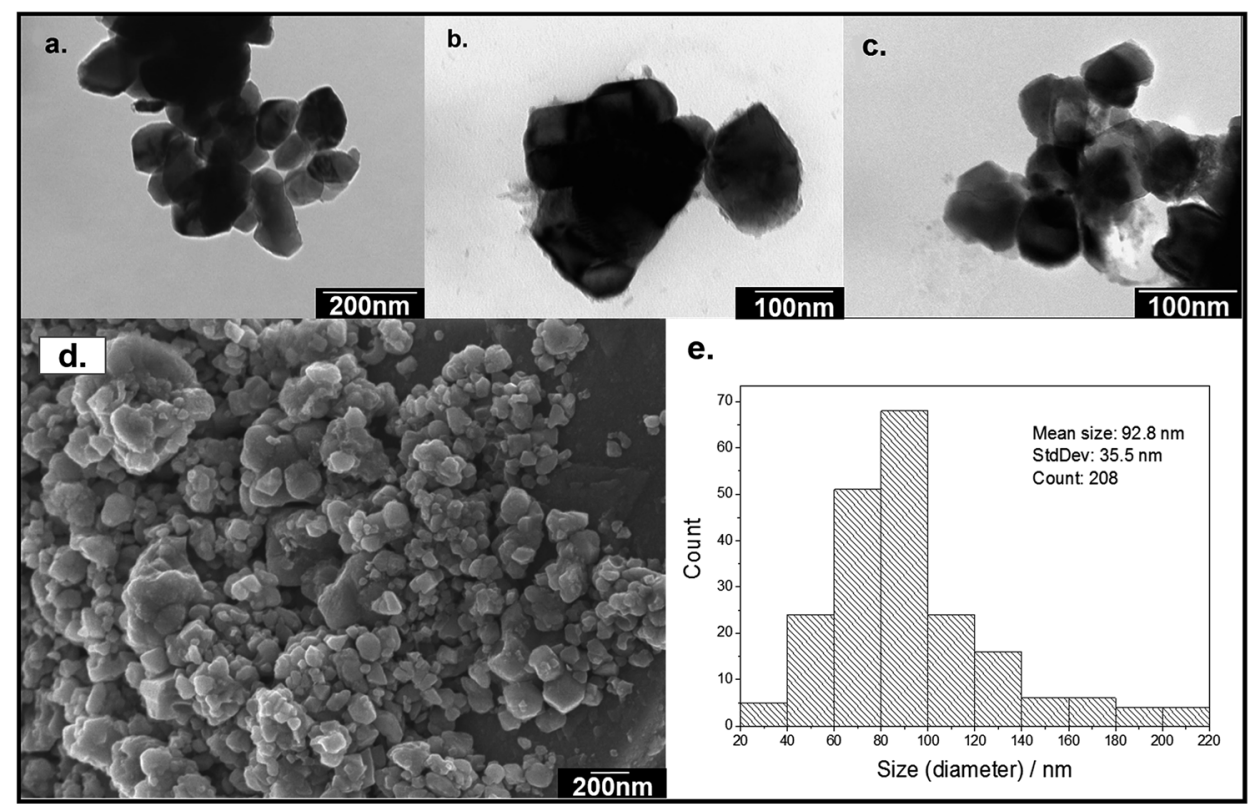

Fig. 4 TEM images of samples: (a) u25\%_24 h_s; (b) u25\%_48 h_s; (c) u25\%_96 h_s. Image (d) is a zoom-out FEG-SEM image and (e) is a histogram showing the particle size distribution. 


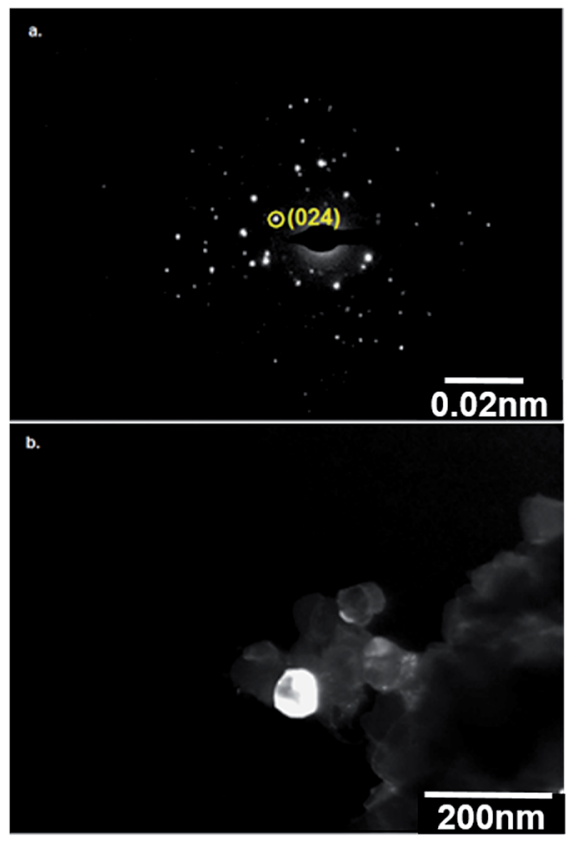

Fig. 5 (a) Electron diffraction pattern and (b) DF-TEM image of u25\% _96 h_s.

characteristic of a few agglomerated single crystals. The DF image shows a single crystal identified as hematite by the (024) plane in ED, which confirms the individual formation of separate nanoparticles of hematite and magnetite.

From the results presented, we can speculate that the synthesis process is governed by the Ostwald step rule. The small amount of goethite detected by Mössbauer spectroscopy is most likely the first step in crystallization from solution, followed by the conversion to hematite, whose formation can be observed after a few hours of solvothermal treatment. Magnetite formation is the last step in phase conversion because magnetite has a lower free energy of formation under standard conditions $^{31}$ and is even more stable under alkaline reducing conditions. The yields of magnetite increased significantly with the solvothermal treatment time at temperatures above $200^{\circ} \mathrm{C}$; however, Pinna et al. noted that temperature higher than $175^{\circ} \mathrm{C}$ favored the formation of hematite..$^{20}$ This contradiction is due to the differences in the mechanisms governing the formation of magnetite in each approach. The formation of magnetite from the reaction between iron(III) acetylacetonate and benzyl alcohol is only possible due to the partial reduction of the metallic center to iron(II), made viable by the oxidation of 4-phenyl2butanone, which produced during the synthesis.

In order to analyze the magnetic properties of these materials, at room temperature $(300 \mathrm{~K})$, vibrating sample magnetometry (VSM) was applied to selected samples, as shown in Fig. 6. Despite the effective amounts of $\mathrm{Fe}_{3} \mathrm{O}_{4}$ and the deviation in particle sizes, as observed in Fig. 4, the magnetic profile was not strongly affected between the samples. The saturation magnetization was around 23 to $37 \mathrm{emu} \mathrm{g}^{-1}$ to all samples, as showed in Table 2, which is comparable to observed by Morel et al. ${ }^{32}$ and $\mathrm{Xu}$ et al. ${ }^{33}$ for particles around the same size distribution. Also, the remanence magnetization, coercitivity and hysteresis indicate a permanent magnetization behavior, but close to a superparamagnetic profile. These results show the applicability of these materials for magnetic purposes.

To investigate the role of the solvent as a capping material, we performed specific experiments to analyze the organic products formed over the particles. Fig. 7 displays the FTIR spectra of benzyl alcohol (BA), used as the solvent; benzyl alcohol after the solvothermal process, obtained as a byproduct (separated by centrifugation in the $\mathrm{Fe}_{3} \mathrm{O}_{4}$ synthesis done in 96
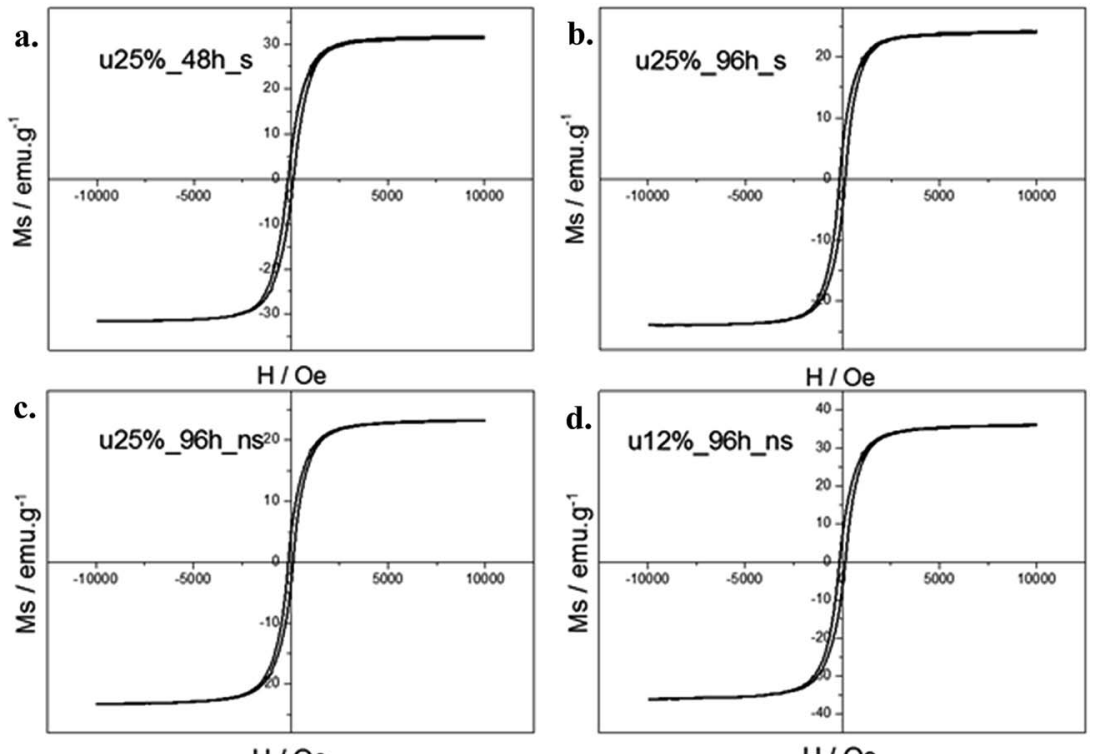

$\mathrm{H} / \mathrm{Oe}$

$\mathrm{H} / \mathrm{Oe}$

Fig. 6 Hysteresis loops obtained by VSM at room temperature for selected samples: (a) u25\%_48 h_s; (b) u25\%_96 h_s; (c) u25\%_96 h_ns and (d) u12\%_96 h_ns. 
Table 2 The saturation magnetization (Ms), remanence (Mr), coercivity (Ce) and hysteresis (Hist) for selected samples obtained by VSM

\begin{tabular}{llllr}
\hline & Ms $\left(\mathrm{emu} \mathrm{g}^{-1}\right)$ & Mr $\left(\mathrm{emu} \mathrm{g}^{-1}\right)$ & Ce (Oe) & Hist $\left(\mathrm{emu} \mathrm{g}^{-1}\right)$ \\
\hline u25\%_48 h_s & 31.7 & 4.9 & 138.2 & 284.9 \\
u25\%_96 h_s & 24.1 & 4.8 & 147.2 & 283.9 \\
u25\%_96 h_ns & 23.2 & 3.9 & 122.4 & 245.5 \\
u12\%_96 h_ns & 36.1 & 6.7 & 152.3 & 299.4
\end{tabular}

hours); and the nanoparticles synthesized over periods of 24,48 and 96 hours. The FTIR spectra of the benzyl alcohol before and after the solvothermal process show similar peaks assigned to the $\mathrm{C}-\mathrm{H}$ stretching of the aromatic ring from 3100 to $3000 \mathrm{~cm}^{-1}$, out-of-plane aromatic $\mathrm{C}-\mathrm{H}$ bending at $726, \mathrm{C}-\mathrm{C}$ ring stretching at 1494 and $1452 \mathrm{~cm}^{-1}$ and ring $\mathrm{C}-\mathrm{C}$ bending at $698 \mathrm{~cm}^{-1}$. The peaks assigned to $\mathrm{O}-\mathrm{H}$ bending at $1208 \mathrm{~cm}^{-1}$ and to $\mathrm{C}-\mathrm{O}$

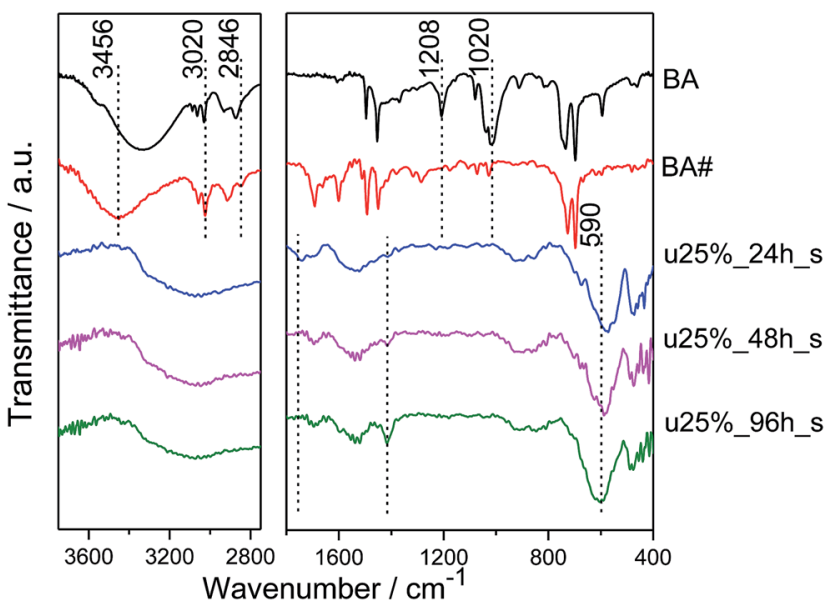

Fig. 7 FTIR spectra of benzyl alcohol (BA), benzyl alcohol after the solvothermal process (BA\#) and the particles synthesized in 24 hours (u25\%_24 h_s), 48 hours (u25\%_48 h_s) and 96 hours (u25\%_96 h_s). stretching at $1020 \mathrm{~cm}^{-1}$ nearly disappeared in the spectrum of benzyl alcohol after the solvothermal process, indicating a reduction in the number of $\mathrm{O}-\mathrm{H}$ groups as the primary alcohol. This result is an indicative of possible benzyl alcohol polymerization. The characteristic intermolecular $\mathrm{O}-\mathrm{H}$ stretching band centered at $3456 \mathrm{~cm}^{-1}$ remained in the spectrum but was less intense and shifted to higher wavelengths due the decrease in the concentration of alcoholic hydroxyl groups. Methylene $\mathrm{C}-\mathrm{H}$ stretching peaks appeared at 2916 and $2846 \mathrm{~cm}^{-1}$, shifted to shorter wavelengths relative to the same peaks in the benzyl alcohol spectrum.

The spectra of particles synthesized in 24,48 and 96 hours, obtained by the diffuse reflectance mode, also showed the peaks of organic residues on the particles' surface forming an overlapping band of the peaks observed between 1770 and 1380 $\mathrm{cm}^{-1}$ in the spectrum of the polymerized benzyl alcohol, related to $\mathrm{C}-\mathrm{C}$ ring stretching, whose shifts were due to the interaction with the particles' surface. At $1415 \mathrm{~cm}^{-1}$, a peak can be observed only in the spectra of the particles synthesized in 48 and 96 hours. The presence of this peak, assigned to $\mathrm{C}-\mathrm{C}$ ring stretching, suggests increasingly effective bonding of the organic residues to the particles as the time of solvothermal treatment and the polymerization degree increased. Additionally, the spectra exhibit the characteristic magnetite band of $\mathrm{Fe}-$ $O$ centered at approximately $590 \mathrm{~cm}^{-1}$. It can be observed that the band is displaced to shorter wavelengths according to the<smiles>Cc1cccc(CO)c1</smiles><smiles>CC(O)(O)COc1ccccc1</smiles><smiles>[O-]c1ccccc1</smiles><smiles>CO[Ge]([O])(O)[Hg]</smiles><smiles>OCc1ccccc1</smiles><smiles>ClCc1ccccc1</smiles><smiles>CCC(C)C</smiles>

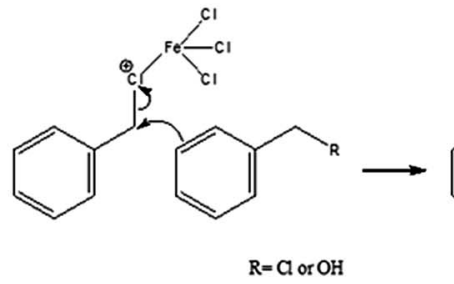

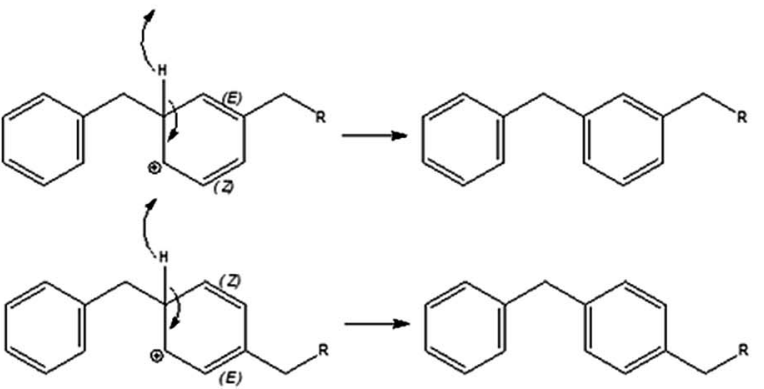

Scheme 1 Reaction mechanism for the polymerization of benzyl alcohol during the solvothermal treatment. 
hematite concentration in the phase mixture because the main characteristic $\mathrm{Fe}-\mathrm{O}$ band for this phase is centered at $550 \mathrm{~cm}^{-1}$.

As cited, during the solvothermal treatment, a possible polymerization of benzyl alcohol occurred, leading to the formation of a dark and sticky supernatant with water release. This process can be associated with the Friedel-Crafts alkylation reaction because $\mathrm{FeCl}_{3} \cdot 6 \mathrm{H}_{2} \mathrm{O}$ is a catalyst for this reaction and benzyl alcohol is susceptible to the reaction. ${ }^{34}$ A possible mechanism for the reaction is shown in Scheme 1. The first step is the reaction of alkyl halide elimination, leading to the formation of benzyl chloride and $\mathrm{Fe}-\mathrm{OH}$ species, which represents the starting point of nanoparticle formation. We considered the direct elimination of benzyl chloride instead of iron alkoxo-halide formation because benzyl groups are able to stabilize a positive charge as a stable carbocation for the SN1 reaction..$^{11,35,36}$

A polymerization reaction follows the formation of benzyl chloride, which reacts with other benzyl chloride or benzyl alcohol molecules, following the Friedel-Crafts mechanism. Benzyl chloride reacts with the catalyst by the attachment of the chlorine atom of the former, creating a more electrophilic carbon on the methylene group. The $\pi$ electrons on the aromatic rings of a benzyl chloride or a benzyl alcohol molecule act as a nucleophile, attacking the electrophilic carbon. Proton removal from $\mathrm{sp}^{3}$ carbon reforms the aromatic ring and leads to the formation of $\mathrm{HCl}$ and catalyst regeneration. The mechanism drawn shows the second step as an SN2 reaction, although it could be an SN1 reaction because the benzylic cation is stable. Friedel and Crafts were the first to observe the polymerization of benzyl chloride, followed by Jacobson, ${ }^{37}$ Dermer and Hooper, ${ }^{38}$ who reported the formation of a soluble, dark-brown resin with the empirical formula $\mathrm{C}_{7} \mathrm{H}_{6}$ as the main reaction product of iron chloride and benzyl chloride. The polymer formed during the synthesis and the mechanism proposed are in agreement with the observations of the aforementioned researchers and with the corresponding FTIR spectrum, which maintains the bands assigned to the aromatic ring but not the bands related to the hydroxyl groups. Furthermore, the polymerization of benzyl alcohol during the synthesis of metal oxide nanoparticles was previously reported by Olliges-Stadler et al. ${ }^{39}$ who obtained a hard and brittle, dark-green-blue monolith with the same empirical formula, but through a different mechanism, while synthesizing tungsten oxide nanoparticles. Differences in the viscosity and color of this polymer are related to the nature of the metallic catalyst, which can lead to different degrees of polymerization. ${ }^{38}$

The polymerization of benzyl alcohol was also verified by nuclear magnetic resonance (NMR) spectroscopy (Fig. 8) using the pure benzyl alcohol (BA) as reference and a sample of the polymerized solvent separated by centrifugation (BApol).

In the BA spectrum, characteristic peaks due to the three different chemical environments are observed: aromatic ring (7.2-7.4 ppm), methylene group (4.6 ppm) and alcohol group (1.8 ppm). The peak assigned to the hydrogen atom of the $\mathrm{OH}$ group (1.8 ppm) in the BA ${ }^{1} \mathrm{H}$-NMR spectrum is suppressed in the spectrum of BApol, which confirms the condensation reaction with water release. The spectrum of BApol displays

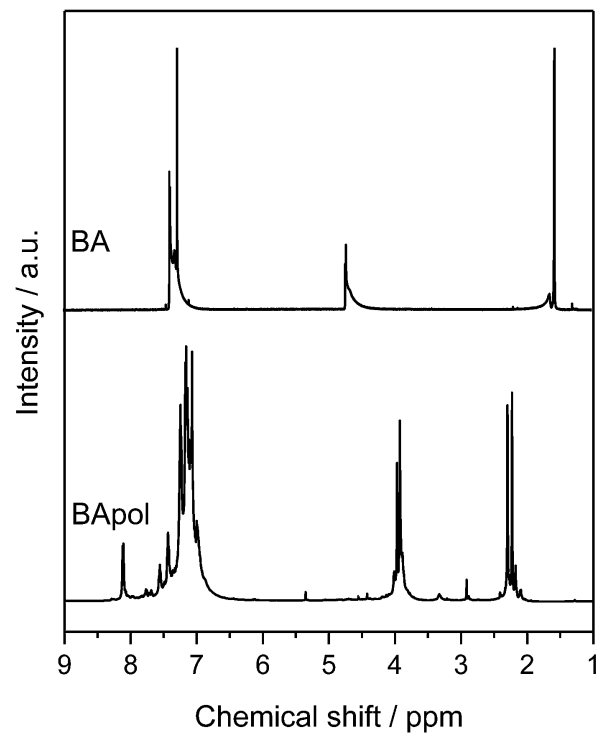

Fig. $8{ }^{1} \mathrm{H}$-NMR spectra of benzyl alcohol (BA) and polymerized benzyl alcohol (BApol).

broad and partly split peaks in three main regions correspondent to the protons of the aromatic rings (6.9-7.6 ppm); to the protons of the aliphatic methylene groups (3.8-4.1 ppm), displaced and outspread from the original positions due to the multiplicity of chemical environments after polymerization; and to the primary hydrogen atoms at terminations (2.1-2.4 ppm).

The polymerization reaction was noticed to be a parallel process to the phase evolution of iron oxides. It occurred independently of the phases obtained and its extent was function of the amount of $\mathrm{FeCl}_{3} \cdot 6 \mathrm{H}_{2} \mathrm{O}$, the Friedel-Crafts catalyst, and solvothermal treatment period. Even though it is not related to the phase evolution, the polymerization of benzyl alcohol has a synergic effect in the global process by capping the as-synthesized magnetite particles and preventing their oxidation for long term stability. Experiments performed in the same samples, stored in dry form during 1 year showed the same results observed for fresh materials, indicating that good stability.

\section{Conclusions}

Based on the results presented herein, we can conclude that magnetite formation under solvothermal conditions follows the Ostwald step rule (from the formation of metastable to that of more stable phases). Goethite formation is most likely the first step in the synthesis process, followed by hematite formation. Metallic iron most likely interacts with the surface of the particles formed, reducing part of the $\mathrm{Fe}^{\mathrm{III}}$ in the crystal structure of the particles to $\mathrm{Fe}^{\mathrm{II}}$, yielding the $\mathrm{Fe}_{3} \mathrm{O}_{4}$ stoichiometry. This hypothesis is consistent with the findings of Matthews, who studied, in detail, magnetite formation under hydrothermal conditions from previously prepared hematite particles through reduction by metallic iron and observed the occurrence 
of two simultaneous processes: oxidation of metallic iron and reduction of hematite to magnetite. ${ }^{40}$

\section{Acknowledgements}

The authors acknowledge the financial support from the Brazilian Agencies CNPq, CAPES, FAPESP, FINEP and Embrapa (Agronano Network). The authors also acknowledge the technical support from LCE-DEMa/UFSCar (for TEM facility), GSMDF/UFSCar (VSM facility) and LIEC/UFSCar (FTIR facility).

\section{Notes and references}

1 U. Jeong, X. W. Teng, Y. Wang, H. Yang and Y. N. Xia, Adv. Mater., 2007, 19, 33-60.

2 S. Laurent, D. Forge, M. Port, A. Roch, C. Robic, L. V. Elst and R. N. Muller, Chem. Rev., 2010, 110, 2574.

3 A.-H. Lu, E. L. Salabas and F. Schüth, Angew. Chem., Int. Ed., 2007, 46, 1222-1244.

4 A. Hu, G. T. Yee and W. Lin, J. Am. Chem. Soc., 2005, 127, 12486-12487.

5 J. Jordan, C. S. S. R. Kumar and C. Theegala, J. Mol. Catal. B: Enzym., 2011, 68, 139-146.

6 D.-H. Chen and M.-H. Liao, J. Mol. Catal. B: Enzym., 2002, 16, 283-291.

7 A. Garcia, S. Oh and C. R. Engler, Biotechnol. Bioeng., 1989, 33, 321-326.

8 I. M. Grabs, C. Bradtmoller, D. Menzel and G. Garnweitner, Cryst. Growth Des., 2012, 12, 1469-1475.

9 P. Hu, L. J. Yu, A. H. Zuo, C. Y. Guo and F. L. Yuan, J. Phys. Chem. C, 2009, 113, 900-906.

10 M. Niederberger, G. Garnweitner, J. Buha, J. Polleux, J. H. Ba and N. Pinna, J. Sol-Gel Sci. Technol., 2006, 40, 259-266.

11 M. Niederberger and N. Pinna, Metal Oxide Nanoparticles in Organic Solvents: Synthesis, Formation, Assembly and Application, Springer, London, 2009.

12 G. Garnweitner and M. Niederberger, J. Am. Ceram. Soc., 2006, 89, 1801-1808.

13 W. Cai and J. Q. Wan, J. Colloid Interface Sci., 2007, 305, 366370.

14 T. Hyeon, S. S. Lee, J. Park, Y. Chung and H. Bin Na, J. Am. Chem. Soc., 2001, 123, 12798-12801.

15 D. Maity, S. N. Kale, R. Kaul-Ghanekar, J. M. Xue and J. Ding, J. Magn. Magn. Mater., 2009, 321, 3093-3098.

16 J. Park, K. J. An, Y. S. Hwang, J. G. Park, H. J. Noh, J. Y. Kim, J. H. Park, N. M. Hwang and T. Hyeon, Nat. Mater., 2004, 3, 891-895.
17 S. Si, C. Li, X. Wang, D. Yu, Q. Peng and Y. Li, Cryst. Growth Des., 2005, 5, 391-393.

18 D. B. Yu, X. Q. Sun, J. W. Zou, Z. R. Wang, F. Wang and K. Tang, J. Phys. Chem. B, 2006, 110, 21667-21671.

19 G. Z. Li, W. C. Peng, X. Y. Li, X. B. Fan, X. J. Li, G. L. Zhang and F. B. Zhang, Appl. Surf. Sci., 2008, 254, 4970-4979.

20 N. Pinna, S. Grancharov, P. Beato, P. Bonville, M. Antonietti and M. Niederberger, Chem. Mater., 2005, 17, 3044-3049.

21 N. Pinna, J. Mater. Chem., 2007, 17, 2769-2774.

22 N. Pinna, M. Karmaoui and M. G. Willinger, J. Sol-Gel Sci. Technol., 2011, 57, 323-329.

23 Y. Masubuchi, S. Yamashita, T. Motohashi, S. Kikkawa and M. Niederberger, J. Eur. Ceram. Soc., 2011, 31, 2471-2474.

24 M. Niederberger, M. H. Bartl and G. D. Stucky, J. Am. Chem. Soc., 2002, 124, 13642-13643.

25 M. Niederberger, M. H. Bartl and G. D. Stucky, Chem. Mater., 2002, 14, 4364-4370.

26 B. H. Toby, J. Appl. Crystallogr., 2001, 34, 210-213.

27 S. Chaleawlert-umpon and N. Pimpha, Mater. Chem. Phys., 2012, 135, 1-5.

28 S. Y. Lian, E. Wang, Z. H. Kang, Y. P. Bai, L. Gao, M. Jiang, C. W. Hu and L. Xu, Solid State Commun., 2004, 129, 485-490.

29 I. J. Bruce, J. Taylor, M. Todd, M. J. Davies, E. Borioni, C. Sangregorio and T. Sen, J. Magn. Magn. Mater., 2004, 284, 145-160.

30 Y. Tamaura, P. V. Buduan and T. Katsura, J. Chem. Soc., Dalton Trans., 1981, 1807-1811.

31 A. Navrotsky, Proc. Natl. Acad. Sci. U. S. A., 2004, 101, 1209612101.

32 M. Morel, F. Martinez and E. Mosquera, J. Magn. Magn. Mater., 2013, 343, 76-81.

33 J. Xu, H. B. Yang, W. Y. Fu, K. Du, Y. M. Sui, J. J. Chen, Y. Zeng, M. H. Li and G. Zou, J. Magn. Magn. Mater., 2007, 309, 307-311.

34 I. Iovel, K. Mertins, J. Kischel, A. Zapf and M. Beller, Angew. Chem., Int. Ed., 2005, 44, 3913-3917.

35 M. Niederberger and G. Garnweitner, Chem.-Eur. J., 2006, 12, 7282-7302.

36 A. Vioux, Chem. Mater., 1997, 9, 2292-2299.

37 R. A. Jacobson, J. Am. Chem. Soc., 1932, 54, 1513-1518.

38 O. C. Dermer and E. Hooper, J. Am. Chem. Soc., 1941, 63, 3525-3526.

39 I. Olliges-Stadler, M. D. Rossell and M. Niederberger, Small, 2010, 6, 960-966.

40 A. Matthews, Am. Mineral., 1976, 61, 927-932. 